\title{
Design and Development of Automatic and Remotely Controlled Plant Watering and Soil Moisture Sensing System
}

\author{
R. Shasikala ${ }^{1}$, M. V. Lakshmaiah ${ }^{2}$ \\ ${ }^{1}$ P.hd Degree in Department of Physics in Rayalaseema University Kurnool, Andhra Pradesh, India \\ ${ }^{2}$ P.hd Degree in Department of Physics in Rayalaseema University Kurnool, Andhra Pradesh, India
}

\begin{abstract}
Agriculture is the backbone of any Country's economy and there is a strong correlation between agricultural growth and economic prosperity. We need a new and effective technology which can improve continuously the productivity, profitability, sustainability of our major farming systems. The main objective of the present paper is to develop a smart wireless sensor network (WSN) for a water management system which is composed of a number of sensor nodes with mesh networking capability that can be deployed for continuous monitoring purpose. The parameters involved in the water management system such as soil moisture, temperature and humidity is measured in the real time by the sensors that send the data to the main controller board. A traditional approach to measure these factors in an agricultural environment meant individuals manually taking measurements and checking them at various times. This paper investigates an automatic irrigation system that switches a motor upon sensing the parameters using Wireless Zigbee Networks and status can be monitored and controlled through GSM based android application. The advantage of using this method is to reduce human intervention and still ensure proper irrigation. The developed platform is cost-effective and allows easy customization.
\end{abstract}

Keywords: ARM Cortex-M3 microcontroller, Zigbee Wireless Networks, Mesh Networks, GSM, Android, PIC Microcontroller, CortexM3, Motor, LCD Display, Soil Moisture, Humidity and Temperature sensors

\section{Introduction}

The need for intelligent farming especially in developing countries like India has grown to a greater extent. Moreover, research in area of Zigbee based wireless sensor network in agriculture, such as monitoring of environmental conditions like weather, soil moisture content, temperature and monitoring growth of the crop, precision agriculture, automated irrigation facility has taken a new dimension.

The rising demands for crop production and quality have significantly increased the utilization of high quality and productivity of green house. The system which we proposed helps us to closely monitor and control the microclimatic parameters of a greenhouse environment. The system comprises of sensors, microcontroller with inbuilt Analog to Digital Convertor (ADC) and actuators (motors, led). When any of the climatic parameters cross a safety threshold which has to be maintained to protect the crops, the sensors sense the change and the client microcontroller reads this from the data at its input ports after being converted to a digital form by the ADC and sends data to main controller through Zigbee network, main controller collects data from several nodes. The main controller then performs the required actions by employing relays until the strayed-out parameter has been brought back to its most advantageous level. Zigbee wireless sensor network takes a major part for connecting multiple nodes to main controller and this process is completely automatic. This system can be controlled manually by Android Smartphone using GSM/GPRS, here it can be implemented either with sms mode or GPRS data packet mode. Not only controlling can get status of climatic conditions and nodes information's to smart phone.
This paper gives a review of controlling and monitoring systems based on existing technologies and a ZigBee based automatic remote control and monitoring and also controlling using GSM based ANDROID application by the user for automatic irrigation system. The design presented has the advantage of ZigBee technology to reduce human intervention and still ensure proper irrigation.

\section{Existing System}

In existing system, automatic controlling process takes place by fully based on single sensor with microcontroller circuit. In other case, it is done by ZigBee wireless network, where ZigBee wireless network is used here for transferring information to the control room which is far away from environment. There the person can control according to their wish and transfer the information via ZigBee connected to controller and according to that performance will takes place. Major Drawbacks

- Collecting samples from one point and judging soil moisture and climatic conditions is not practically feasible.

- In microcontroller based circuit-The actions which we need to perform at any time cannot be done in any case

- Existing systems are designed with 8-bit microcontroller.

- In ZigBee wireless network based controlling process all the information stored in microcontroller is send to PC which is far away in control room through ZigBee transceiver. The person in control room will perform control action. According to that microcontroller will actuate the actuators. 


\section{International Journal of Science and Research (IJSR) \\ ISSN (Online): 2319-7064}

Index Copernicus Value (2013): 6.14 | Impact Factor (2015): 6.391

\section{Proposed System}

An attractive thing of proposed system is that, it has extension in its concepts in a successful way. Here both automatic controlling (microcontroller \& Zigbee based) and remotely controlling (Android application based) takes place. Here Main controller is powerful 32bit ARM Cortex-M3 (multi task execution).Automatic controlling process takes place continuously with multiple clients connected to main controller in Zigbee wireless sensor network which automatically switches the motor and also farmer can know the status of the system on his mobile app like humidity, soil moisture content, temperature, motor status and from which time motor has been running. The communication between main controller and android phone is GSM/GPRS.

Software Tools:

- Orcad Capture 10.0.

- Cadstar13

- Proteus for simulation.

- MPLAB IDE(xc8 compiler).

- Keil uvusion 5 Mdk-ARM.

- Android Studio.

\section{Wireless Sensor Networks}

A WSN is a system comprised of radio frequency (RF) transceivers, sensors, microcontrollers and power sources. Recent advances in wireless sensor networking technology have led to the development of low cost, low power, multifunctional sensor nodes. Sensor nodes enable environment sensing together with data processing. Instrumented with a variety of sensors, such as temperature, humidity and volatile compound detection, allow monitoring of different environments. They are able to form network with other sensor systems and exchange data with external users. Sensor networks are used for a variety of applications, including wireless data acquisition, machine monitoring and maintenance, smart buildings and highways, environmental monitoring, site security, automated on-site tracking of expensive materials, safety management, and in many other areas. A general WSN protocol consists of the application layer, transport layer, network layer, data link layer, physical layer, power management plane, mobility management plane and the task management plane. Currently two standard technologies are available for WSN: ZigBee and Bluetooth. Both operate within the Industrial Scientific and Medical (ISM) band of $2.4 \mathrm{GHz}$, which provides license free operations, huge spectrum allocation and worldwide compatibility. In general, as frequency increases, bandwidth increases allowing for higher data rates but power requirements are also higher and transmission distance is considerably shorter. Multihop communication over the ISM band might well be possible in WSN since it consumes less power than traditional single hop communication.

\subsection{Overview of Zigbee Technology}

ZigBee is the name of a specification for a suite of high level communication protocols using small, low-power digital radios based on the IEEE 802.15.4 standard for wireless personal area networks (WPANs). ZigBee is targeted at RF applications that require a low data rate, long battery life, and secure networking.

ZigBee operates in the industrial, scientific and medical (ISM) radio bands; $868 \mathrm{MHz}$ in Europe, $915 \mathrm{MHz}$ in the USA and $2.4 \mathrm{GHz}$ in most jurisdictions worldwide. The technology is intended to be simpler and cheaper than other WPANs such as Bluetooth.

\subsection{Architecture}

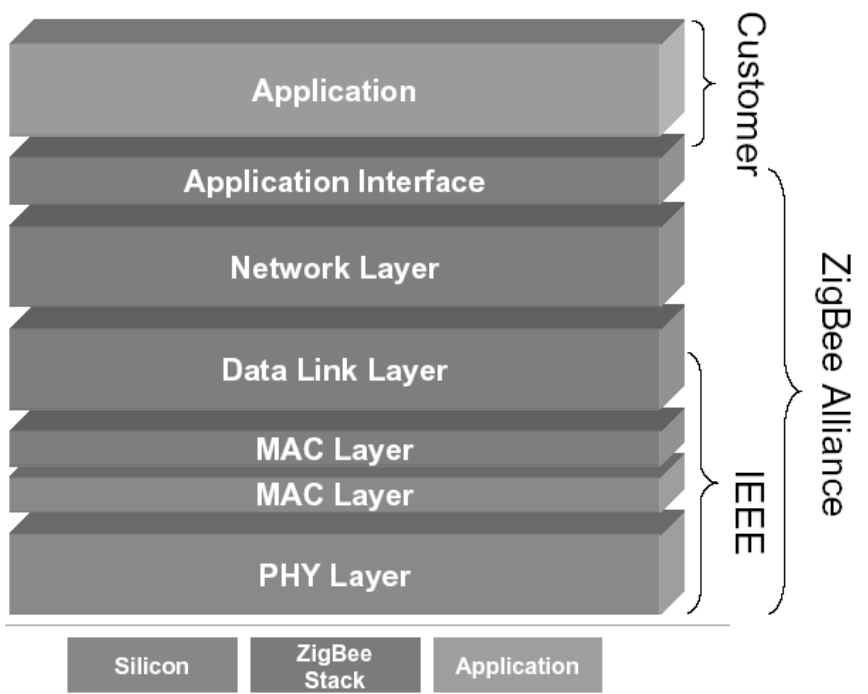

Figure 1: Architecture

\subsubsection{ZigBee/IEEE 802.15.4- General Characteristics}

- Dual PHY (2.4GHz and $868 / 915 \mathrm{MHz})$.

- Data rates :250 kbps @ $2.4 \mathrm{GHz}, 40$ kbps @ $915 \mathrm{MHz}, 20$ kbps@868 MHz

- Optimized for low duty-cycle applications $(<0.1 \%)$

- CSMA-CA channel access Yields high throughput and low latency for low duty cycle devices like sensors and controls

- Low power (battery life multi-month to years).

- Multiple topologies Star, Tree, Mesh.

- Addressing space of up to $18,450,000,000,000,000,000$ devices (64 bit IEEE address)and 65,535 networks

- Optional guaranteed time slot for applications requiring low latency

- Fully hand-shaked protocol for transfer reliability

- Range: $10 \mathrm{~m}-1 \mathrm{Km}$

\subsubsection{Physical Layer}

The very first layer is Physical Layer and this is designed by IEEE. There are two Physical layers (PHY), both phys use Direct Sequence Spread Spectrum (DSSS). The modulation type in the $2.4 \mathrm{GHz}$ band is O-QPSK with a $32 \mathrm{PN}$-code length and an RF bandwidth of $2 \mathrm{MHz}$. 


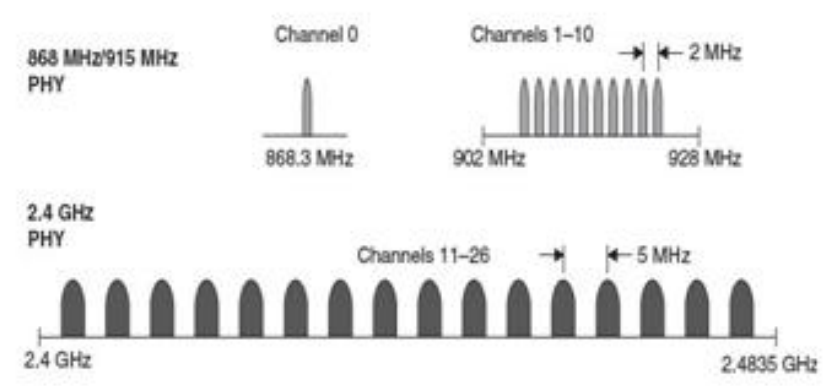

Figure 2:

Functions of Physical Layer

- Energy detection (ED).

- Link quality indication (LQI).

- Channel selection

- Clear channel assessment (CLA)

\subsubsection{MAC Layer}

The main function of MAC is to carry out the association and disassociation of the network involved a large number of devices are managed or handled by this layer. Employs 64bit IEEE \& 16-bit short addresses Using local addressing $65,000\left(2^{\wedge} 16\right)$ nodes can be configured, with reduced address overhead. Three devices employed

(a) Network Coordinator

(b) Full Function Device/Router (FFD)

(c) Reduced Function Device/End Device (RFD)

\section{Functions of MAC Layer:}

- It generates the network beacons according to the device, if it is a coordinator

- It also performs the function of synchronizing the beacons.

- It uses the CSMA-CA channel access mechanism

- It uses Guaranteed Time Slot (GTS) mechanism.

- It allows different mechanisms to conserve energy like collision avoidance using CSMA-CA and allowing the device to go into sleep mode.

\section{(a) Network Coordinator}

Maintains overall network knowledge and it also initiates the network, most sophisticated of the three types; most memory and computing power, all devices will communicate with this and it also act as a bridge to the other networking . Zigbee Network has unique Personal Area Network(PAN)ID and channel number.

\section{(b) Full Function Device:}

Carries full 802.15.4 functionality and all features specified by the standard Additional memory, computing power make it ideal for a network router function it manages local address allocation/de-allocation and it can work in any topology.

\section{(c) Reduced Function Device:}

Carries limited (as specified by the standard) functionality to control cost and complexity General usage will be in network edge devices, it is limited to work in STAR topology. All of these devices can be no more complicated than the transceiver, a simple 8-bit MCU and a pair of AAA batteries.

\subsection{Zigbee Topologies}

ZigBee supports several network topologies; however, the most commonly used configurations are star, mesh and cluster tree topologies. Any topology consists of one or more coordinator. In a star topology, the network consists of one coordinator which is responsible for initiating and managing the devices over the network. All other devices are called end devices that directly communicate with coordinator. This is used in industries where all the end point devices are needed to communicate with the central controller, and this topology is simple and easy to deploy.

In mesh and tree topologies, the Zigbee network is extended with several routers where coordinator is responsible for staring them. These structures allow any device to communicate with any other adjacent node for providing redundancy to the data. If any node fails, the information is routed automatically to other device by these topologies. As the redundancy is the main factor in industries, hence mesh topology is mostly used. In a cluster-tree network, each cluster consists of a coordinator with leaf nodes, and these coordinators are connected to parent coordinator which initiates the entire network.

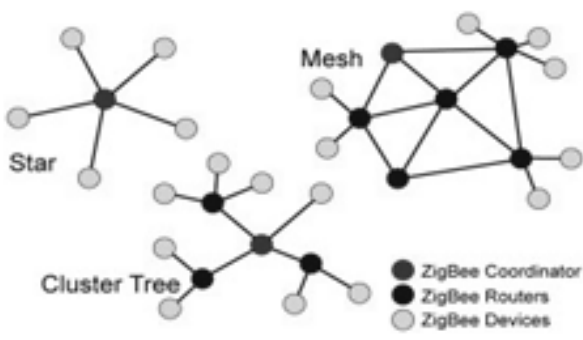

Figure 3: ZigBee addresses three typical traffic types. IEEE 802.15.4 MAC can accommodate all the types.

1)Data is periodic. Application dictates the rate, and the sensor activates checks for data and deactivates.

2)Data is intermittent. Application, or other stimulus, determines the rate, as in the case of say smoke detectors. The device needs to connect to the network only when communication is necessitated. This type enables optimum saving on energy.

3)Data is repetitive, and the rate is fixed a priori. Depending on allotted time slots, called GTS (guaranteed time slot), devices operate for fixed durations.

ZigBee employs either of two modes, beacon or non-beacon to enable the to-and-fro data traffic. Beacon mode is used when the coordinator runs on batteries and thus offers maximum power savings, whereas the non-beacon mode finds favor when the coordinator is mains-powered.

In the beacon mode, a device watches out for the coordinator's beacon that gets transmitted at periodically, locks on and looks for messages addressed to it. If message transmission is complete, the coordinator dictates a schedule for the next beacon so that the device 'goes to sleep'; in fact, the coordinator itself switches to sleep mode.

While using the beaconmode, all the devices in a mesh network know when to communicate with each other. In this mode, necessarily, the timing circuits have to be quite

Volume 6 Issue 1, January 2017

www.jijs.net

Licensed Under Creative Commons Attribution CC BY 


\section{International Journal of Science and Research (IJSR) \\ ISSN (Online): 2319-7064}

Index Copernicus Value (2013): 6.14 | Impact Factor (2015): 6.391

accurate, or wake up sooner to be sure not to miss the beacon. This in turn means an increase in power consumption by the coordinator's receiver, entailing an optimal increase in costs.

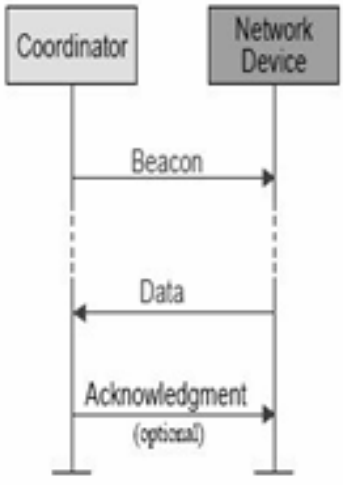

Figure 4: Beacon Network Communication

The non-beacon mode will be included in a system where devices are 'asleep' nearly always, as in smoke detectors and burglar alarms. The devices wake up and confirm their continued presence in the network at random intervals. On detection of activity, the sensors 'spring to attention', as it were, and transmit to the ever-waiting coordinator's receiver (since it is mains-powered).

However, there is the remotest of However, there is the remotest of chances that a sensor finds the channel busy, in which case the receiver unfortunately would 'miss a call'.

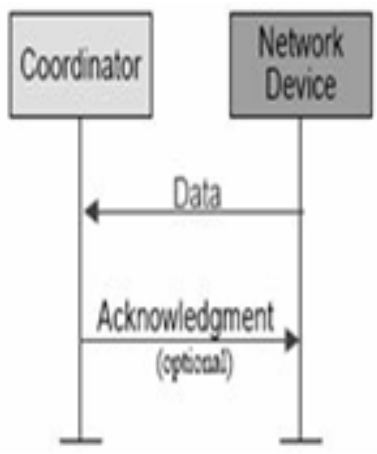

Figure 5: Non-Beacon Network Communication

\begin{tabular}{|c|c|c|c|}
\hline \multicolumn{4}{|c|}{ Existing Wireless technologies comparison } \\
\hline Parameter & ZigBee & Bluetooth & Wi-Fi \\
\hline Range & $10 \mathrm{~m}-100 \mathrm{~m}$ & $1 \mathrm{~m}-10 \mathrm{~m}$ & $1 \mathrm{~m}-100 \mathrm{~m}$ \\
\hline $\begin{array}{l}\text { Operating } \\
\text { Frequency }\end{array}$ & $\begin{array}{c}2.4 \mathrm{GHz} \text { globally, } 915 \mathrm{MHz} \\
\text { - USA, 868MHz -Europe }\end{array}$ & $\begin{array}{l}2.4 \mathrm{GHz} \\
\text { globally }\end{array}$ & $\begin{array}{l}2.4 \mathrm{GHz} \& \\
5 \mathrm{GHz}\end{array}$ \\
\hline $\begin{array}{l}\text { Peak Data } \\
\text { Rate }\end{array}$ & $\begin{array}{l}250 \mathrm{Kbps} @ 2.4 \mathrm{GHz} \\
40 \mathrm{Kbps} @ 915 \mathrm{MHz} \\
20 \mathrm{Kbps} @ 868 \mathrm{MHz}\end{array}$ & $\begin{array}{l}1 \mathrm{Mbps} / \\
3 \mathrm{Mbps}\end{array}$ & $\begin{array}{c}11 \mathrm{Mbps} \\
@ 2.4 \\
\mathrm{GHz} \\
54 \mathrm{Mbps} \\
\text { @ } 5 \mathrm{GHz}\end{array}$ \\
\hline Modulation & DSSS & $\begin{array}{l}\text { Adaptive } \\
\text { FHSS }\end{array}$ & DSSS \\
\hline $\begin{array}{l}\text { No: of } \\
\text { physical } \\
\text { layers }\end{array}$ & $2(2.4 \mathrm{GHz}, 915 / 868 \mathrm{MHz})$ & $\begin{array}{c}1 \mathrm{PHY} 2.4 \\
\text { GHz }\end{array}$ & $\begin{array}{c}1 \text { PHY } 2.4 \\
\text { GHz }\end{array}$ \\
\hline $\begin{array}{l}\text { Networks } \\
\text { supported }\end{array}$ & Star, mesh, cluster tree & $\begin{array}{l}\text { Point to } \\
\text { point, } \\
\text { Point to } \\
\text { multipoint, }\end{array}$ & $\begin{array}{l}\text { Peer to } \\
\text { Peer, IP }\end{array}$ \\
\hline
\end{tabular}

\begin{tabular}{|c|c|c|c|}
\hline & & $\begin{array}{l}\text { Peer to } \\
\text { Peer. }\end{array}$ & \\
\hline $\begin{array}{l}\text { Device } \\
\text { types }\end{array}$ & $\begin{array}{c}\text { Coordinator, Router, End } \\
\text { device }\end{array}$ & $\begin{array}{l}\text { Master, } \\
\text { Slaves }\end{array}$ & $\begin{array}{l}\text { Access } \\
\text { point and } \\
\text { STA }\end{array}$ \\
\hline $\begin{array}{c}\text { Total } \\
\text { number of } \\
\text { devices } \\
\text { networks }\end{array}$ & $\begin{array}{c}\text { With 64-bit IEEE address } \\
18,450,000,000,000,000,000 \\
\text { devices can be addressed } \\
\text { and } 16 \text {-bit short addressing, } \\
\text { 65,535 nodes and } 255 \\
\text { devices per network }\end{array}$ & 8 & $\begin{array}{c}32 / \text { Access } \\
\text { point }\end{array}$ \\
\hline Latency & $30 \mathrm{msec}$ & upto $10 \mathrm{sec}$ & $\begin{array}{c}\text { Upto } 10 \\
\text { sec }\end{array}$ \\
\hline $\begin{array}{l}\text { Total No: } \\
\text { of channels }\end{array}$ & $\begin{array}{r}16 @ 2.4 \mathrm{GHz}, \\
10 @ 915 \mathrm{MHz}, \\
1 @ 868 \mathrm{MHz}\end{array}$ & 79 & 14-Nov \\
\hline $\begin{array}{l}\text { Protocol } \\
\text { stack }\end{array}$ & 4-32 KB typical & $\sim 100 \mathrm{~KB}$ & $\sim 100 \mathrm{~KB}$ \\
\hline $\begin{array}{c}\text { Channel } \\
\text { access } \\
\text { mechanism }\end{array}$ & CSMA/CA & Poll-Reply & CSMA/CA \\
\hline Bandwidth & $250 \mathrm{Kbps}$ & $1 \mathrm{Mbps}$ & $22 \mathrm{Mbps}$ \\
\hline
\end{tabular}

\section{Proposed System}

An attractive thing of proposed system is that, it has extension in its concepts in a successful way. Here both automatic controlling (microcontroller \& Zigbee based) and manual controlling (Android application based) takes place. The proposed system design includes a powerful 32bit ARM Cortex-M3 (multi task execution) microcontroller, PIC 16F microcontroller, Zigbee modules, GSM modem, Temperature, humidity, soil moisture sensors. Here system comprises of Local controller board (client Transmitter node) and the Main controller board.

Multiple soil moisture sensors are deployed in the field at various locations in a Zigbee wireless network which are configure to form a mesh network so as to measure the volumetric water content in soil. Each installed sensor has its identifier pertaining to the Farming field. The client sensor node examines the information at regular intervals send collected data to the main controller board over Zigbee wireless networks which in turn operate the motor based on collected information.

As the sensor node captures parameters like temperature, soil moisture and humidity are sent to the main controller and these parameters can be monitored and controlled on mobile with android app through GSM remotely, this can be achieved by either sms mode or GPRS packet mode.Thisdata can be used for precision farming and actuators can be controlled using microcontroller data. This is how total working takes place of automation of irrigation system. Different sensors like moisture and temperature sensor senses the moisture content and temperature required. Thus it helps to provide a proper environment to grow crops easily.

i) We have designed Zigbee wireless sensor mesh network for monitoring the crop field area by deploying moisture sensors in the land to detect the places where the water level is low. As the system is designed in mesh network it can be up scaled in future without much effort.

ii) We used humidity sensor to sense the weather. By this the farmer can get idea about the climate. If there is any chance for rainfall, there is no need to pump water to the

Volume 6 Issue 1, January 2017 


\section{International Journal of Science and Research (IJSR) \\ ISSN (Online): 2319-7064}

Index Copernicus Value (2013): 6.14 | Impact Factor (2015): 6.391

crop field. Due to this we can conserve water and also power since we didn't turn on motors. iii) System can be controlled and monitored over GSM so the farmer or controller need not to go and switch $\mathrm{ON}$ the mains.

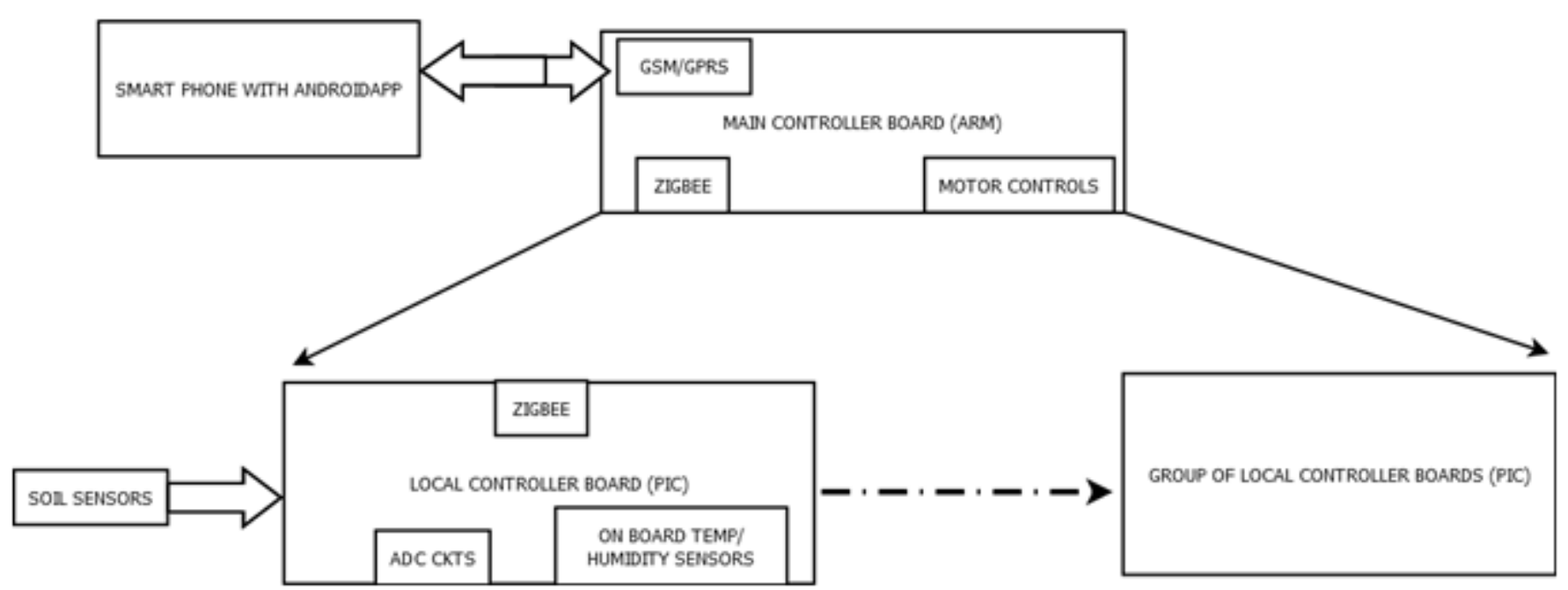

Figure 6: Main Block Diagram

\subsection{Main Controller Board- Receiver}

This board includes ARM controller, GSM modem, zigbee module, battery and motor controls. It receives information from the client nodes and processes the data and controls the motor operation. And also status can be shown on the mobile through android application using GSM/GPRS.

\subsubsection{ARM-Cortex M3 Microcontroller}

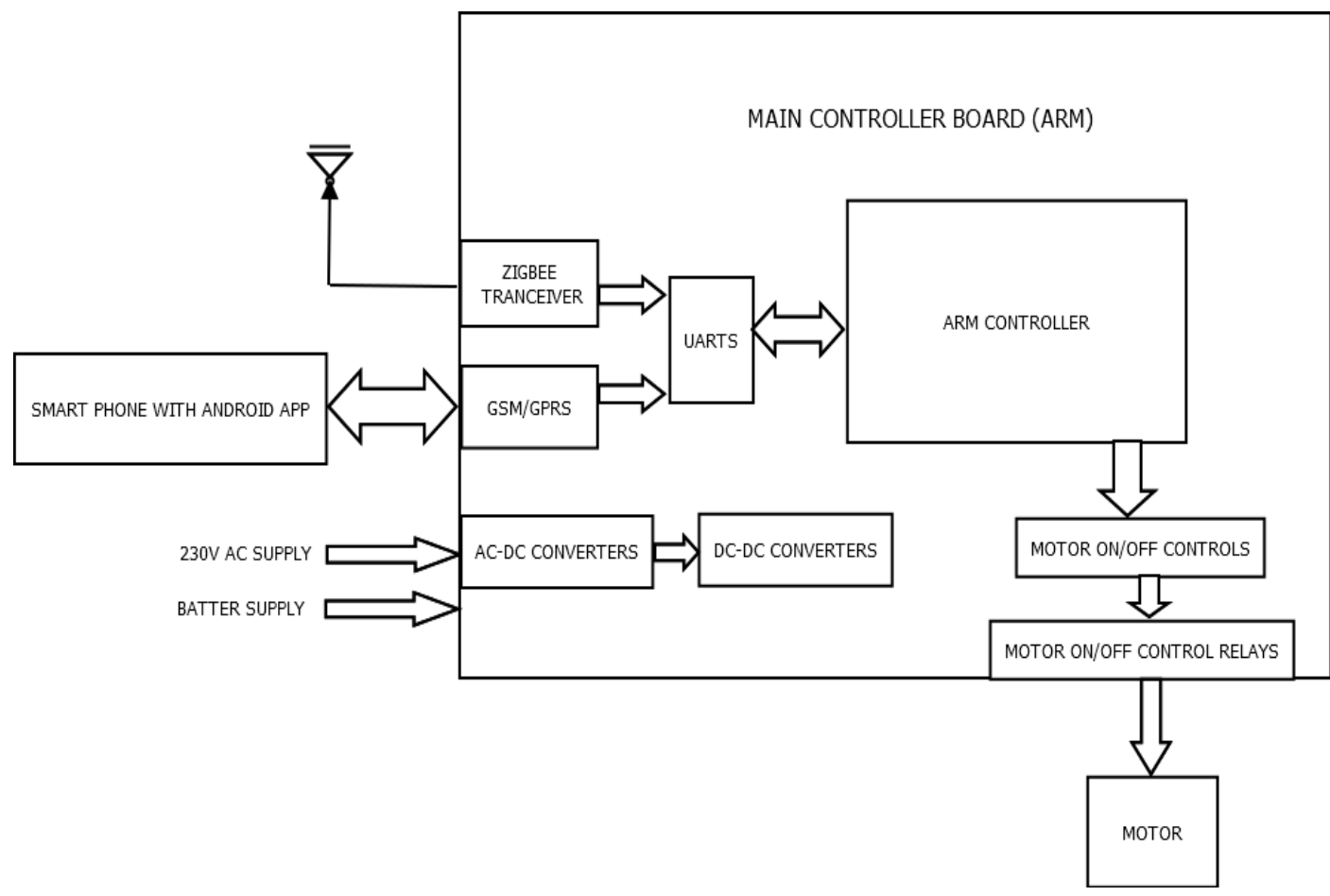

Advanced RISC Machine (ARM) is a family of reduced instruction set computing (RISC) architectures for computer processors, configured for various environments. ARM is a systems-on-chip (SoC) that incorporate memory, interfaces, radios, etc.

Volume 6 Issue 1, January 2017 


\section{International Journal of Science and Research (IJSR) \\ ISSN (Online): 2319-7064}

Index Copernicus Value (2013): 6.14 | Impact Factor (2015): 6.391

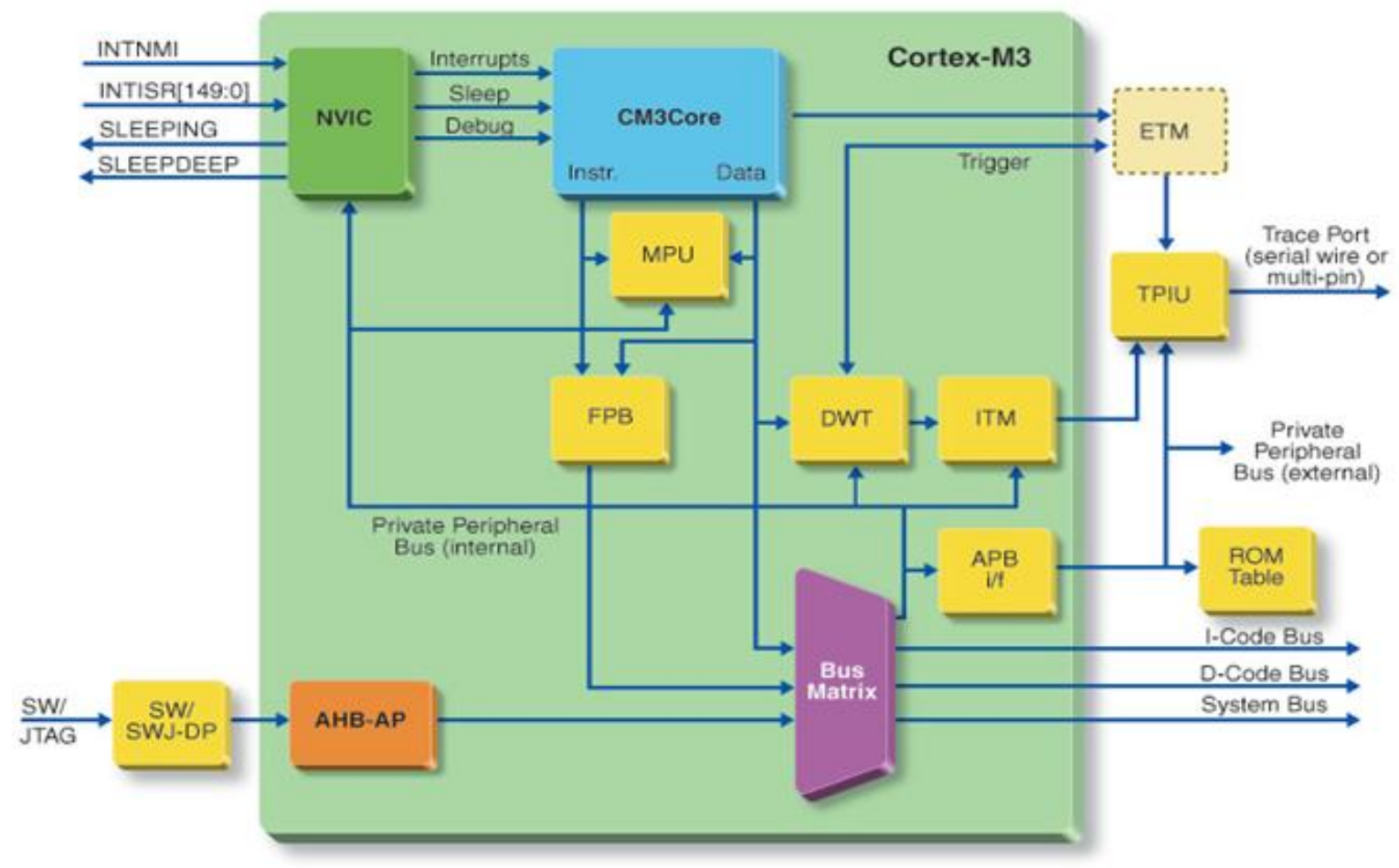

Figure 7: MainController Board

As per our requirement in this project Cortex-M3 controller is chosen.

Key features of the Cortex-M3 core are:

- ARMv7-M architecture

- 18 x 32-bit registers

- Reduced pin count requirements

- Efficient interrupt handling

- Power management

- Efficient debug and development support features

- Breakpoints, Watchpoints,

- Flash Patch support,

- Instruction Trace

- User/Supervisor model

- OS support features

- Designed to be fully programmed in C (even reset, interrupts ).

\subsubsection{Zigbee Module}

The XBee RF Modules are designed to operate within the Zigbee protocol and support the unique needs of low-cost, low-power wireless sensor networks. The modules require minimal power and provide reliable delivery of data between remote devices. The modules operate within the ISM 2.4 $\mathrm{GHz}$ frequency band. It operates over a range of 100- 200 meters. Beside figure shows the Zigbee module.

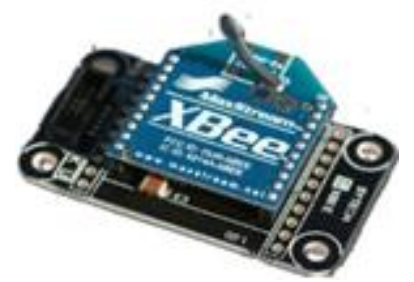

\subsubsection{SIM900A}

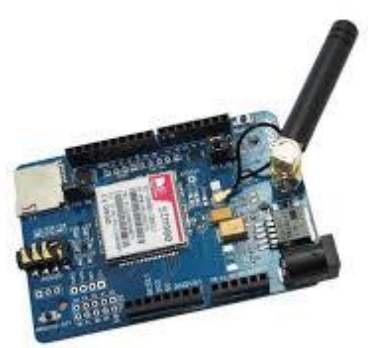

GSM Sim 900A GSM/GPRS Modem-RS232 is built with Dual Band GSM/GPRS engine- SIM900A, works on frequencies 900/ $1800 \mathrm{MHz}$. The Modem is coming with RS232 interface, which allows you connect PC as well as microcontroller with RS232 Chip(MAX232). The baud rate is configurable from 9600-115200 through AT command. The GSM/GPRS Modem is having internal TCP/IP stack to enable you to connect with internet via GPRS. It is suitable for SMS, Voice as well as DATA transfer application in M2M interface. The onboard Regulated Power supply allows you to connect wide range unregulated power supply . Using this modem, you can make audio calls, SMS, Read SMS, attend the incoming calls and internet ect through simple AT commands.

\subsection{Wireless Sensor Nodes}

Sensor nodes have different sensors like humidity sensor, temperature sensor and soil moisture sensor senses moisture content and sends the data to main controller board over Zigbee network.

\subsubsection{Microcontroller}

Microcontrollers are used in automatically controlled products and devices, such as automobile engine control systems, implantable medical devices, remote controls,

\section{Volume 6 Issue 1, January 2017 www.ijsr.net}




\section{International Journal of Science and Research (IJSR) \\ ISSN (Online): 2319-7064 \\ Index Copernicus Value (2013): 6.14 | Impact Factor (2015): 6.391}

office machines, appliances, power tools, toys and other embedded systems. The Proposed System Uses PIC 16F873 Microcontroller. PIC16F873 features 256 bytes of EEPROM data memory, self programming, an ICD, 8 channels of 10bit Analog-to-Digital (A/D) converter, 2 capture/compare/PWM functions, the synchronous serial port can be configured as either 3-wire Serial Peripheral Interface (SPI) or the 2-wire InterIntegrated Circuit $\left(\mathrm{I}^{2} \mathrm{C}\right)$ bus and a Universal Asynchronous Receiver Transmitter (USART) [6]. In this proposed system microcontroller will take the analog data from different sensor which will gets converted into digital one with the help of ADC present in microcontroller. After conversion completion the data will be displayed on LCD and will be given to Zigbee for wireless transmission.

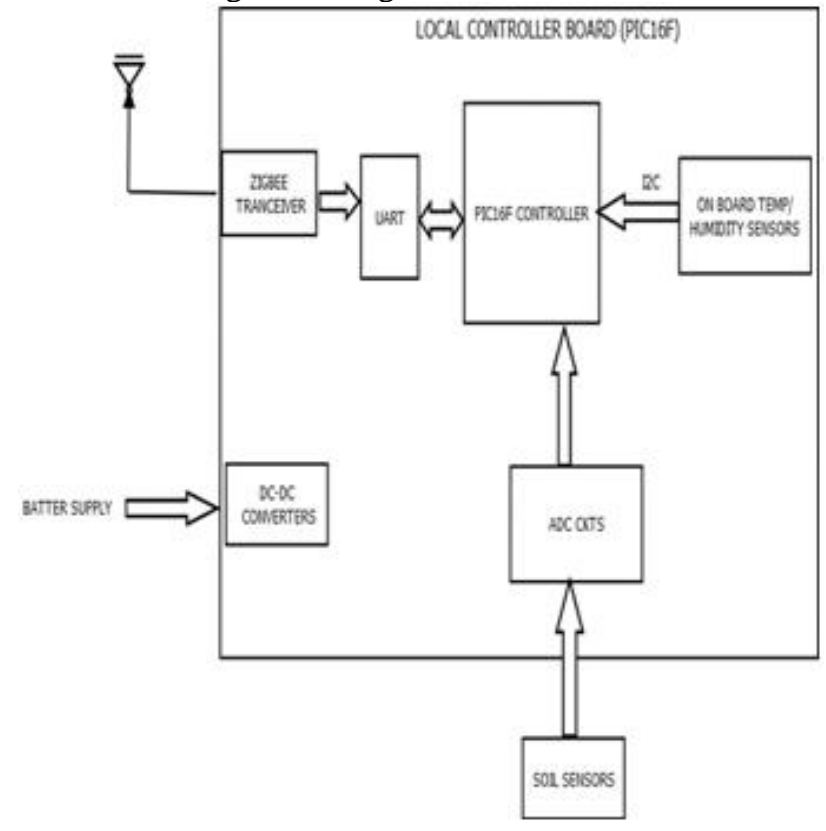

\subsubsection{Sensor}

Sensor is a device which is used to convert physical quantity into electrical signal. A sensor is a device, which responds to an input quantity by generating a functionally related output usually in the form of an electrical or optical signal.

Sensors used to measure environmental parameters are

- Temperature sensor (LM35)

- Humidity sensor

- Soil Moisture Sensor

\section{a) Temperature Sensor}

The LM35 is an integrated circuit sensor that can be used to measure temperature with an electrical output proportional to the temperature (in oC). It is used to measure temperature more accurately than a using a thermistor. The sensor circuitry is sealed and not subject to oxidation. The LM35 generates a higher output voltage than thermocouples and may not require that the output voltage be amplified. This sensor is used to monitor surrounding temperature. It gives the idea about the increase or decrease in the temperature of surrounding. If the temperature changes it is observed on LCD.

\section{b)Humidity Sensor}

Humidity is an expression of the amount of water vapour in air. It is an invisible gas that varies between $1-4 \%$ of our atmosphere by volume. SYHS-220 sensor module converts relative humidity $(30-90 \% \mathrm{RH})$ to voltage and can be used in weather monitoring application. This sensor is used to monitor humidity i.e. moisture present in the surrounding.

\section{c) Soil moisture sensor}

Soil moisture sensor measures the content of water present in the soil. It works on the principle of frequency domain reflectometry technique of soil moisture measurement. Frequency domain reflectometry uses capacitance probes to measure the moisture. The moisture is calculated by considering the dielectric constant of the soil. The dielectric constant of any material is defined as capacity to transmit the electromagnetic pulses or waves. The dielectric constant of water is much bigger in value than soil. Two electrodes are embedded into the soil and soil acts as dielectric medium. The electrodes are given voltage supply, due to presence of water the dielectric of soil changes. Because of which the frequency oscillations occur, at a certain point resonance occurs and the resonance frequency value is used to calculate the water content in the soil. More the water, smaller will be the resonant frequency. FDR is more accurate method as compared to TDR. It is not affected by the salinity levels, gives better resolution.

\subsubsection{Relay}

A relay is an electrical switch. This electrical switch is operated by an electromagnet that opens and closes under the control of another electrical circuit it is able to control an output circuit of higher power than the input circuit. Relays are used where it is necessary to control a circuit by a lowpower signal or where several circuits must be controlled by one signal.

\subsubsection{Actuation system}

An actuator is a piece of equipment which will produce a movement when signal is given. Actuators are used in the computer control of an environment, industrial automation and in robotics or, more generally, actuators are the machines used for output in control applications. The designed system includes a motor as an actuator for pumping water to the field upon the intruction of microcontroller.

\section{Proposed System}

\subsection{Embedded C}

An embedded system is some combination of computer hardware and software, either fixed in capability or programmable that is specifically designed for a particular function. The software is installed on the controller, the brains of the electronic device. Each embedded system issued for one specific function.

The $\mathrm{C}$ language can also use very simple commands to control the device, freeing up the limited memory of the device to hold many commands or parameters. $\mathrm{C}$ can be written for both microcontrollers and digital signal processors. Code is written in C on a programmer's PC.Code is run through a compiler on the programmer's $\mathrm{PC}$ to create a software program. The embedded system software may be run through a simulator on the programmer's computer. The software program is copied onto the controller using a 


\section{International Journal of Science and Research (IJSR) \\ ISSN (Online): 2319-7064}

Index Copernicus Value (2013): 6.14 | Impact Factor (2015): 6.391

"programmer." The controller is then tested on a "test bed" to ensure that it works properly.

Programming in $\mathrm{C}$ makes the Embedded systems more reliable. $\mathrm{C}$ code written for a specific micro controller can easily be transferred to systems using different microcontrollers of different vendors without little or no modification. It can be reused, easy to maintain and easy to debug and extend. The advantages of $\mathrm{C}$ as shown in the following:

1) Of higher level languages, $C$ is the closest to assembly languages.

2) Most micro controllers have available $C$ compilers.

3)Writing in $\mathrm{C}$ simplifies code development for large projects.

4)It is easier and less time consuming to write in $C$ language than assembly.

5) $\mathrm{C}$ is easier to modify and update.

6) We can use code available in function libraries.

7) C code is portable to other micro controllers with little or no change.

8) Assembly is the fastest, however, it is difficult to find or train assembly experts. Then if a new processor is required, we have to start over!

9) $\mathrm{C}$ is mid-level, lots of good $\mathrm{C}$ programmers are available, $\mathrm{C}$ compilers are available. $\mathrm{C}$ can be used on 8-, 16-, 32-, and 64-bits processors.

\subsection{Cadence OrCAD}

Cadence OrCADPCB Designer with PSpice comprises three main applications.

- Capture - used to drawn a circuit on the screen, known formally as schematic capture. It offers great flexibility compared with a traditional pencil and paper drawing, as design changes can be incorporated and errors corrected quickly and easily. (On the other hand, it is much faster to develop the outline of a circuit using pencil and paper.)

- PSpice - simulates the captured circuit. You can analyse its behavior in many ways and confirm that it performs as specified.

- PCB Editor - for the design of printed circuit boards. The output is a set of files that can be sent to a manufacturer or the electronics workshop in the Rankine Building. I do not cover PCBs in this handout. The programs communicate using files called netlists.

\subsection{Proteus for Simulation}

It is a software suite containing schematic, simulation as well as PCB designing.

ISIS is the software used to draw schematics and simulate the circuits in real time. The simulation allows human access during run time, thus providing real time simulation.

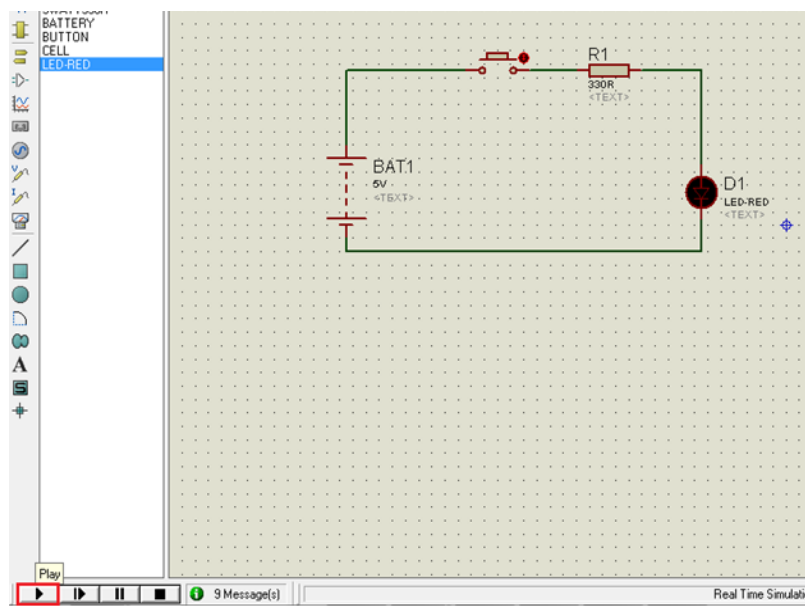

Connecting the circuit,click on the play button to run the simulation

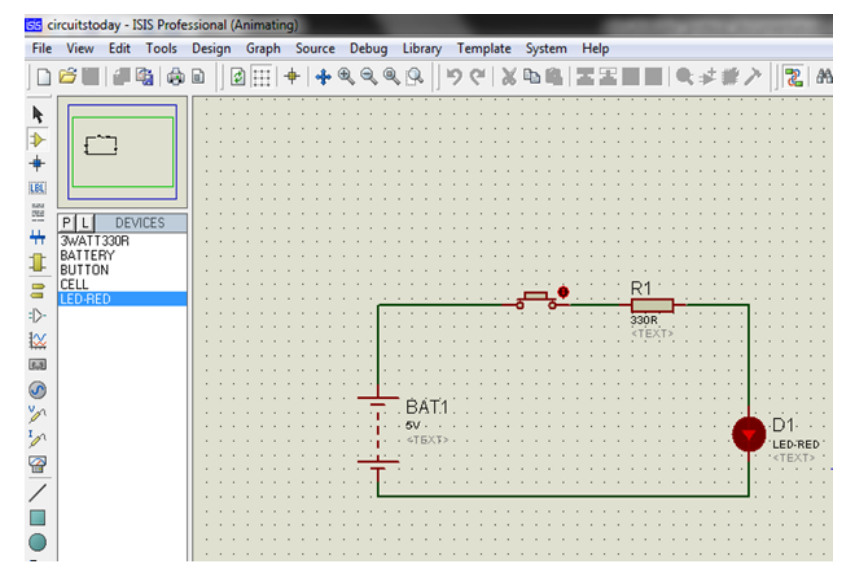

The button is depressed during simulation by clicking on it to make LED glow

\subsection{MPLAB IDE XC8}

It is a proprietary freeware integrated development environment for the development of embedded applications on PIC and dsPIC microcontrollers, and is developed by Microchip Technology. MPLAB $®$ XC Compilers produce highly optimized code. $\mathrm{XC} 8$ is a $\mathrm{C}$ compiler for 8-bit PIC devices.

\subsection{Keil uvision 5 Mdk-ARM}

The MDK-ARM is a complete software development environment for Cortex ${ }^{\mathrm{TM}}-\mathrm{M}$, Cortex-R4, ARM7TM and ARM9TM processor-based devices. MDK-ARM is specifically designed for microcontroller applications.

\subsection{Android}

Android is an open source and Linux based operating system for mobile devices such as smartphones and tablet computers. Android was developed by the Open Handset Alliance, led by Google, and other companies.

Android applications are usually developed in the Java language using the Android Software Development Kit. Once developed, Android applications can be packaged easily and sold out either through a store such as Google Play Store. 


\section{International Journal of Science and Research (IJSR) \\ ISSN (Online): 2319-7064}

Index Copernicus Value (2013): 6.14 | Impact Factor (2015): 6.391

\subsection{Android Studio}

Android Studio is the official integrated development environment (IDE) for Android platform development. Features are provided in the current stable version

- Gradle-based build support.

- Android-specific refactoring and quick fixes.

- Lint tools to catch performance, usability, version compatibility and other problems.

- ProGuard integration and app-signing capabilities.

- Template-based wizards to create common Android designs and components.

- A rich layout editor that allows users to drag-and-drop UI components, option to preview layouts on multiple screen configurations

- Support for building Android Wear apps

\section{Conclusion}

Zigbee-based agriculture monitoring system serves as a reliable and efficient system for efficiently monitor the environmental parameters. Wireless monitoring of field not only allows user to reduce the human power, but it also allows user to see accurate changes in it. This research focuses on developing devices and tools to manage, display and alert the weather/disaster warnings using the advantages of a wireless sensor network system.

\section{References}

[1] N. G Shah and I. Das, "Precision Irrigation Sensor Network Based Irrigation", a book on Problems, Perspectives and Challenges of Agricultural Water Management, IIT Bombay, India, pp. 217-232, April 2008.

[2] M. K. Haefke, S. Mukhopadhyay and H. Ewald, "A Zigbee Based Smart Sensing Platform for Monitoring Environmental Parameters", IEEE Conference on Instrumentation and Measurement Technology, pp. 1-8, May 2011.

[3] Q. Wang, A. Terzis and A. Szalay, "A Novel Soil Measuring Wireless Sensor Network", IEEE Transactions on Instrumentation and Measurement, pp. 412-415,August 2010.

[4] I. Singh and M. Bansal, "Monitoring Water Level in Agriculture using Sensor Networks", International Journal of Soft Computing and Engineering, pp. 202204, November 2011.

[5] Dursun, M. \& Ozden, S. (2011). “A wireless application of drip irrigation automation supported by soil moisture sensors" Scientific Research and Essays. 6(7):15731582

[6] Zhou, Y., Yang, X., Wang, L., Ying, Y., 2009. “A wireless design of low-cost irrigation system using ZigBee technology". In: 2009 International Conference on Networks Security, Wireless Communications and Trusted, Computing, pp. 572-575.

[7] Oliveira, L. M. L., Rodrigues, J. J. P. C. (2011), Wireless sensor networks: a survey on environmental monitoring, Journal of Communications, vol. 6, 2, pp. 143-151.

[8] Oliveira, L. M. L., Rodrigues, J. J. P. C. (2011), Wireless sensor networks: a survey on environmental monitoring,
Journal of Communications, vol. 6, 2, pp. 143-151.

[9] Krishnamachari, B. (2005), Networking Wireless Sensors, Cambridge University Press.

[10] Stipanicev, D., Bodrozic, Lj., Stula, M. (2007), Environmental intelligence based on advanced sensor networks, In Proceedings of the 14th International Workshop on 2007 IWSSIP and ECSIPMCS, Maribor, Slovenia.

[11] Android Programming: The Big Nerd Ranch Guide (Big Nerd Ranch Guides) Kindle Edition by Brian Hardy (Author), Bill Phillips (Author)

[12] Programming Android Java Programming for the New Generation of Mobile Devices by Zigurd Mennieks , Laird Dornin, G. Blake Meike ,\& Mausmi Nakamura

[13]Hello, Android Introducing Google's Mobile Development Platform" by Ed Burnette

[14] http://www.onlineprogrammingbooks.com/android/

[15] ARM Limited. Cortex-M3 technical reference manual, 2006.

[16] STMicroelectronics. User manual STM32 value line discovery, 2010.UM0919.

[17] STMicroelectronics. Programming manual: Stm32f10xxx/ 20xxx/21xxx/ 11xxxx cortex-m3 programming manual, March 2011. PM0056.

[18]Embedded C Programming and the Microchip PIC, Volume 1 By Richard H. Barnett, Larry O'Cull, Sarah.

[19] Programming the PIC Microcontroller with MBASIC By Jack R. Smith

[20] https://en.wikibooks.org/wiki/A_Guide_To_PIC_Microc ontroller_Documentation/Documentation_structure 